\title{
Sensor based Prediction of Human Driving Decisions using Feed forward Neural Networks for Intelligent Vehicles
}

\author{
Shriram C Jugade ${ }^{1}$, Alessandro C Victorino ${ }^{2}$, Véronique B Cherfaoui ${ }^{1}$, Stratis Kanarachos ${ }^{3}$
}

\begin{abstract}
Prediction of human driving decisions is an important aspect of modeling human behavior for the application to Advanced Driver Assistance Systems (ADAS) in the intelligent vehicles. This paper presents a sensor based receding horizon model for the prediction of human driving commands. Human driving decisions are expressed in terms of the vehicle speed and steering wheel angle profiles. Environmental state and human intention are the two major factors influencing the human driving decisions. The environment around the vehicle is perceived using LIDAR sensor. Feature extractor computes the occupancy grid map from the sensor data which is filtered and processed to provide precise and relevant information to the feed-forward neural network. Human intentions can be identified from the past driving decisions and represented in the form of time series data for the neural network. Supervised machine learning is used to train the neural network. Data collection and model validation is performed in the driving simulator using the SCANeR studio software. Simulation results are presented along with the analysis.
\end{abstract}

Index Terms-Intelligent vehicle, ADAS, human driving behavior, human driving decisions, Shared Control, Autonomous Navigation, Neural Networks.

\section{INTRODUCTION}

In the last years, we have faced the need and development of a new class of ADAS modules, taking into account the interaction between the human and the embedded autonomous navigation system. Although, these ADAS include autonomous functionalities enabling the fully control the displacements of the actuated intelligent vehicle, the final control input results from the fusion between the human driver control and the intelligent system control, in a shared navigation strategy ([1]). Predicting the human driving behavior is of main interest in the development and application to these new ADAS modules, making them more efficient and proactive. This is the problem treated in this article. There are several aspects of predicting the human driving behavior. One of the aspects is to identify patterns and classify the driving behavior ([2],[3],[4]). This helps for the automated driving to understand the behavior of human drivers in other vehicles.

Vehicle trajectory prediction is another major aspect of modeling human driving behavior. The prediction of trajec-

This project has received funding from the European Commission under the H2020 Grant agreement ITEAM No. 675999 and by LABEX MS2T, ROBOTEX

${ }^{1}$ Sorbonne universités, Université de Technologie de Compiègne, CNRS, Heudiasyc UMR 7253, CS 60319; 60203 Compigne Cedex, France. Email: shriram.jugade/veronique.cherfaoui @ hds.utc.fr

${ }^{2}$ Department of Mechanical Engineering, Universidade Federal de Minas Gerais (UFMG), Av Antonio Carlos 6627, Belo Horizonte, Minas Gerais, Brazil. Email: avictorino@ufmg.br

${ }^{3}$ Coventry University, United Kingdom. Email: ab8522@ coventry.ac.uk tory is either in generalized form using deep neural networks ([5], [6]) or specific to some scenarios for e.g. lane change ([7]). These trajectory prediction methods are well suited for the behavioral cloning ([8]) or assessment of automated driving ([9]). However, neural networks requires lot of data for the training and validation purpose, and second the knowledge of the future trajectory of the vehicle does not directly provide the information about the vehicle speed and steering profiles. Hence, it is difficult to identify human driving commands. Another related work, with the prediction of vehicle speed and steering wheel angle is presented in [10] which uses deep belief nets and predicts the commands only for one time step in the future.

As mentioned before, our main research objective for the development of the human driving predictive model (presented in this paper) is to apply it for the shared driving control. The presented methodology predicts the human driving decisions in the form of vehicle speed and steering wheel angle with prediction horizon of medium size (4 secs). In order to avoid the problems associated to the deep neural networks (demanding lot of data for the training and validation), we have developed a feature extractor to filter relevant data and process it before sending it to the neural network. We used a driving simulator along with the SCANer studio software for the data collection and validation.

The paper is organized as follows: Section II explain the model architecture in detail including feature extraction, vehicle speed prediction and steering wheel angle prediction. Section III explain the neural network development. This includes the data collection method and process, neural network structural design, outlier detection and elimination method, training and validation etc. Section IV present the simulation results along with analysis. Section V concludes the topic along with an insight into the future works.

\section{Human Driver Model Architecture}

Human driving decisions can be expressed in terms of the vehicle speed and steering wheel angle. These are sufficient to determine the driving behavior and to predict the vehicle trajectory for the future time instants. The presented model will predict the driving command profiles for a particular time horizon based on the environment perception, human intention and current vehicle state. The predictions will be updated at each time sample i.e. the model is of the receding horizon type. The time horizon considered for the vehicle speed profile prediction is 4 secs while that for the steering wheel angle profile is 2 secs. The rapid changes in the steering wheel angle for a given scenario restricts the 
prediction capability of the model to a shorter time horizon. The model is of the open loop form i.e. the predictions of the model at current time instant are not fed back for the next prediction. This allows the model to be dependable on the inputs rather than the past predictions. The main objective of this strategy is to eliminate the error propagation of the past predictions.

The model architecture (shown in Fig. 1) shows different components each having a unique functionality. The main function of the prediction is performed by a multi-layer feedforward neural network. It is trained on the input-output driving data collected separately for the training purpose. The inputs shown in the architecture are selected based on the human approach towards driving decision-making and can be classified into three categories namely: environmental state, vehicle state and the human intention. Past information of the inputs is used to identify the variation with respect to time. This adds a time series perspective to the model.

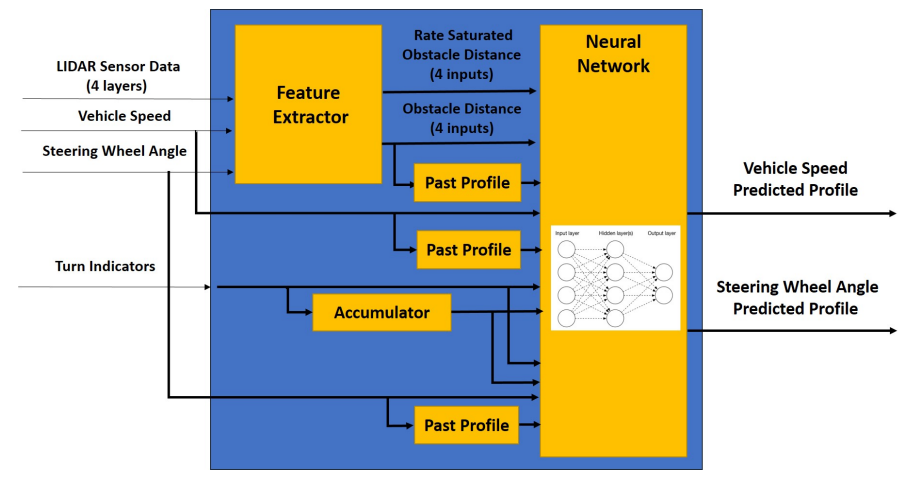

Fig. 1. Model architecture for the prediction of human driving commands

\section{A. Vehicle Speed Profile Prediction}

The environment perception of the human driver can not be captured directly. Hence, we have to rely on sensors for environment perception which may differ a bit from that of the human driver. The model is designed to be robust to such errors/differences. The obstacles are identified using LIDAR sensor and environment perception techniques. The presented modeling methodology uses LIDAR sensor with 4 vertical layers, 90 degrees field of view and has a range of $110 \mathrm{~m}$. The methodology can be extended to any other configuration of LIDAR sensor. The sensor data contains lot of information which needs to be processed and filtered before sending it to the neural network. This function is performed by the feature extractor. It identifies the obstacles relevant to the driving decision, calculates the relative distance to the closest obstacle in a given direction (that is the predicted ahead path for the vehicle: explained later) and processes it further. This processed data is then sent to the neural network.

The human driving decisions are not always dependent on the environmental factors for e.g. the human driver may slow down the vehicle because of a reason other than the state of the obstacles. Such intentions are very hard to predict in some difficult maneuvers but can be identified in the form of time series. Hence, the model uses the past information of the vehicle speed for the predictions. The past input variation information can be represented in the form of the polynomial coefficients obtained through polynomial regression (quadratic). Such a representation reduces the number of inputs of the neural network. The past information of the feature extractor data is also represented in the form of polynomial coefficients. The function for the polynomial regression and extraction of the coefficients is shown as "Past Profile" block in the Fig. 1. For the model, the past input information is limited to 2 secs.

The driving decisions related to the vehicle speed are also affected when the human driver is about to take a turn for e.g. depending on the current vehicle speed and turning radius, the human driver slows down the vehicle before taking a turn. Hence, the turn indicator (left and right) signal is considered to be one of the inputs to the model. The accumulator (shown as Accumulator in Fig. 1) is a sample counter from the instant when the turn indicator is on. This signal is relevant for this model because the human driver does not turn the vehicle immediately after the turn indicator signal is on. Hence, the probability of the human driver taking the turn increases with the accumulation signal. The accumulator value resets after each cycle of the turn indication.

\section{B. Steering Wheel Angle Profile Prediction}

The vehicle steering future decisions are dependent mainly on the road curvature, human intention to take the turn or change the lane or overtake another vehicle. Currently in this model we are not considering the road/lane orientation. The main inputs correlated to the future steering wheel angle decisions are the turn indicators signal, the past values of the steering wheel angle etc. These decisions are partially dependent on the environmental state. Hence, the neural network uses all the inputs to find the correlations.

\section{Feature Extractor}

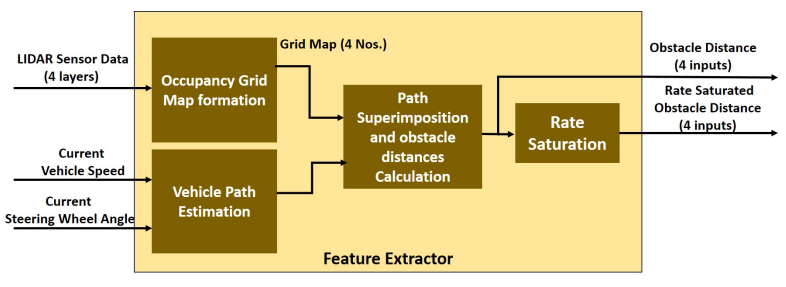

Fig. 2. Block diagram of Feature Extractor

Feature extractor (as shown in Fig. 2) is used for processing the LIDAR sensor data and filtering out relevant information. For the prediction of speed profile, relative distances (between the target vehicle and the obstacles) and their variation with time is of main interest. For e.g. if the target vehicle is approaching a static obstacle (another vehicle, pedestrian etc), the relative distance decreases with time. In case of proper driving, it can be predicted that the human driver will slow down the vehicle to avoid collision. The LIDAR sensor data is used to form a probabilistic 
occupancy grid map (one for each layer of LIDAR). The grid maps contain information related to all the objects and other vehicles in the environment around the target vehicle. With respect to the variation of the vehicle speed, the driving decisions are influenced only by the obstacles present in the path of the vehicle. Thus, not all the information present in the grid maps is relevant to the prediction of the future vehicle speed profile.

The relevant information is filtered out of grid maps by superimposing the estimated path of the vehicle onto the map. The vehicle trajectory in general is considered to be of circular form. Given a time instant, approximate angular velocity of the vehicle is computed from its speed and wheel angle (computed through the steering ratio). The turning radius of the vehicle is computed from the speed and angular velocity and the estimated circular trajectory of the vehicle is superimposed on the grid maps. An example of the grid maps with and without superimposition of the vehicle's estimated path is shown in Fig. 3. The width of the path is approximately equal to the vehicle's width. The intersection of the estimated path and the obstacle present in the grid maps implies that the obstacle is present in the intended path and is thus considered to be relevant. The distance (straight line) between the target vehicle and the relevant obstacle is calculated for each grid map. In the case of multiple intersections, obstacle closest to the target vehicle is considered.

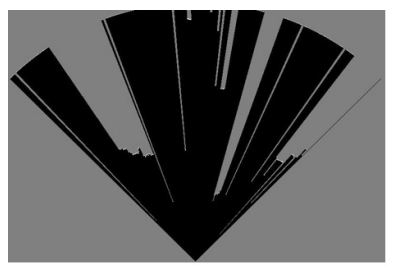

(a)

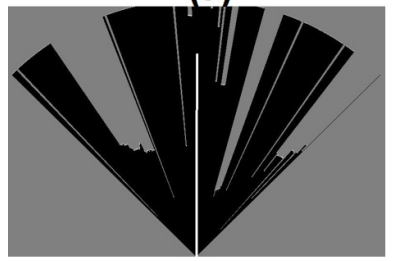

(b)

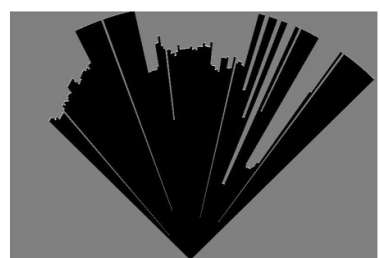

(c)

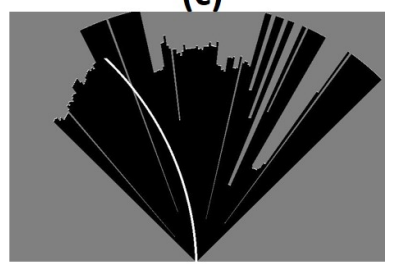

(d)
Fig. 3. Sample Occupancy Grid maps of single layer of LIDAR sensor. (a) and (c) show the probabilistic grid map at different time instants. (b) and (d) are the respective grid maps with the intended vehicle path superimposed on them.

The uncertainties in the sensor data is reflected in the grid map which affects the relative distance profile. Also, the variation in the vehicle's pitch create additional uncertainties in the sensor data and hence in the grid map. These uncertainties give rise to the impulses in the profile of the relative distance (for each grid map). These impulses will affect the training as well as performance of the neural network. The impulses are created mainly due to the false detection of the objects and during quick turns. Rate saturation is applied to the variation of relative distances. This gives the information to the neural network about the impulses. The upper and lower threshold values for the rate saturation are $1000 \mathrm{~m} / \mathrm{s}$ and $10 \mathrm{~m} / \mathrm{s}$. The lower limit of the rate saturation is concerned with the false obstacle detection or to ignore obstacles during the transition phase of quick turns. The upper limit is concerned with the increase in free space in the intended vehicle's path. Hence, the lower rate saturation limit is kept much less as compared to the upper limit. These are selected by trial and error method. An example of the relative distance profile and its rate saturated profile is shown in Fig. 4.

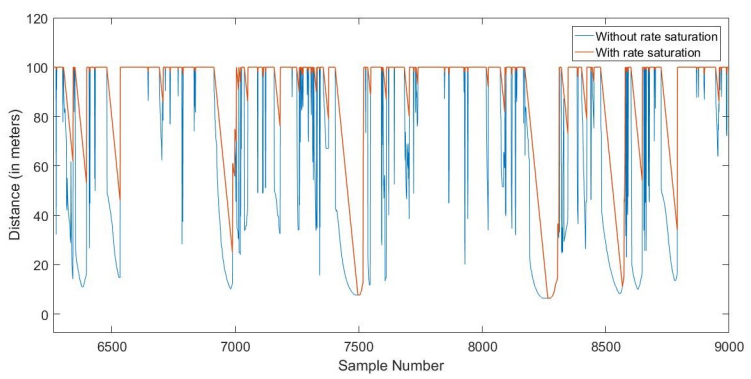

Fig. 4. Relative Distance profile (one LIDAR layer) with and without rate saturation

\section{NeURAL NETWORK DEVElopMENT}

\section{A. Architecture}

As mentioned before, multi-layer feed-forward neural network is used in the model development to predict the vehicle speed and steering wheel angle profiles. The architecture of the neural network is shown in 5. The number of input and outputs are 30 each. The number of future profile samples (output) of vehicle speed and steering wheel angle are 20 and 10 respectively. Because of the complexity and non-linearity of the correlation between inputs and outputs, two hidden layers with 40 neurons each are considered. The number of hidden neurons is based on the number of inputs and outputs. The neural network trained using supervised machine learning techniques. Hence, given the vehicle and environment state (inputs) at a time instant, the neural networks will predict the driving decisions. The neural network is implemented in the Neural Network Toolbox of Matlab.

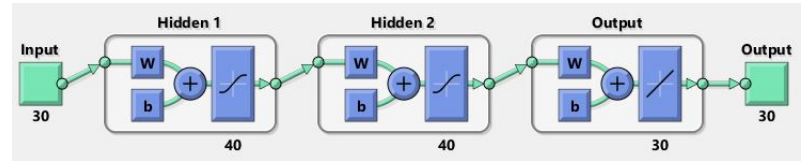

Fig. 5. Neural Network Structure

\section{B. Training}

For the purpose of training and offline validation of the neural networks, data is collected using the driving simulator and SCANeR studio software as shown in Fig. 6 from a city 
map inbuilt in this software. This map has various structures and objects for e.g. buildings, multi-lane roads, trees, bridge etc. The varied scenarios available in the map provides the data close to the real world.

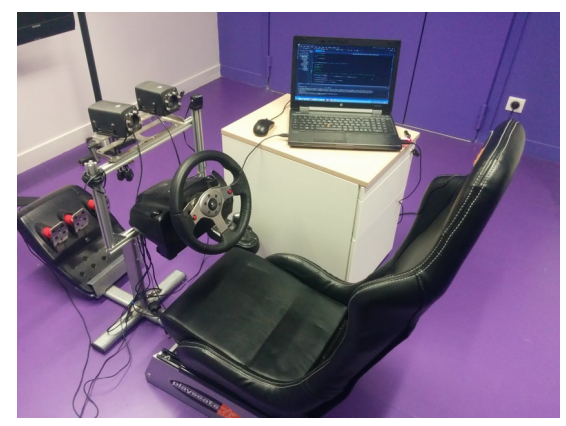

Fig. 6. Driving Simulator Setup

Data is collected at the sampling rate of $5 \mathrm{~Hz}$ for the duration of 1 hour. This choice of sampling rate helps in the removal of impulsive disturbances in the data without lose of any important information. Virtual LIDAR sensor is mounted on the vehicle. The variation in the pitch and roll of the vehicle affects the sensor data, along with the built in disturbances. These factors help in getting sensor data close to that of the sensor in the real world.

Given the vehicle and environment state, the prediction horizon for the vehicle speed and steering wheel angle profiles are 4 and 2 secs respectively. The sharp and consecutive turns lead to the fast variation of the steering wheel angle which makes it difficult to predict for a larger horizon. The steering wheel angle varies mainly during curved roads, lane changes and turns, all of which last for a shorter duration. Labeled data for the training and validation of neural networks are created accordingly. Simulink and SCANeR studio software are integrated for the data collection and validation. Feature extraction algorithm is implemented in Matlab/Simulink which processes the collected data and forms input/output data sets.

The data set is divided into two parts (ratio 2:1) for the purpose of training and validation respectively. LevenbergMarquardt optimization method is used for the training purpose. It is the fastest back propagation algorithm available in the toolbox and gives the best training results when compared to the application of other algorithms. The performance of the neural networks are computed in the form of mean squared error (MSE).

\section{Outlier detection and elimination}

The uncertainties in the data set (for e.g. uncertainties in the LIDAR sensor data) creates outliers, thus affecting the learning performance of the neural networks. A higher threshold is set for the output error to identify the outliers. The training samples identified as outliers using this threshold are assigned a zero weight and the rest are assigned non-zero weight. The neural networks are trained again using weighted MSE as the performance function which ignores the training samples with zero weight. The detection
TABLE I

NeUral Network PERformance

\begin{tabular}{|c|c|c|}
\hline & Speed prediction & Steering angle prediction \\
\hline & MSE $(\mathrm{kmph})$ & MSE $(\mathrm{rad} / \mathrm{sec})$ \\
\hline Training & 5.5 & 0.03 \\
\hline Validation & 8.1 & 1.5 \\
\hline
\end{tabular}

and elimination of outliers improves the neural network performance. The total number of outliers eliminated is kept under $10 \%$ of the training data set. The training and offline performance of both the neural networks are provided in Table I.

\section{Simulation Results And AnAlysis}

The validation using SCANer studio has been carried out for various use cases and the test performance is as given in Table I. We present the results and analysis of three of those use cases.

Use Case 1: This use case is related to the basic collision avoidance. At the start of the use case, the obstacles are dynamic and they come to a halt due to a traffic light. Since the target vehicle is driving in the same lane, the driver applies brakes and stops the vehicle smoothly. The snap shots of the use case are shown in Fig. 7 in a summarized form.

The profile of vehicle speed and brake pedal force is shown in Fig. 8. The brake pedal force is not used by the model and is considered for the analysis of the results only. Since the human driving model uses receding horizon, it is not possible to show the entire prediction in one graph. Hence, for the analysis, critical instants from the use case are considered and shown. In this use case, the driver applies brakes at around 11 secs. It is expected from the model to predict the decrease in the vehicle speed profile (due to obstacles) before the driver applies brakes. This phenomenon is of early prediction is shown in Fig. 10. For the purpose of analysis, we have shown the profile of the relative distances (given as outputs by Feature Extractor) in Fig. 9. The graph clearly shows the decrease in the relative distance between the target vehicle and relevant obstacle due to which an early prediction is possible. The model continues with the correct prediction in the future as shown in Fig. 11. There is no change in the steering angle profile because of which it is not shown here.

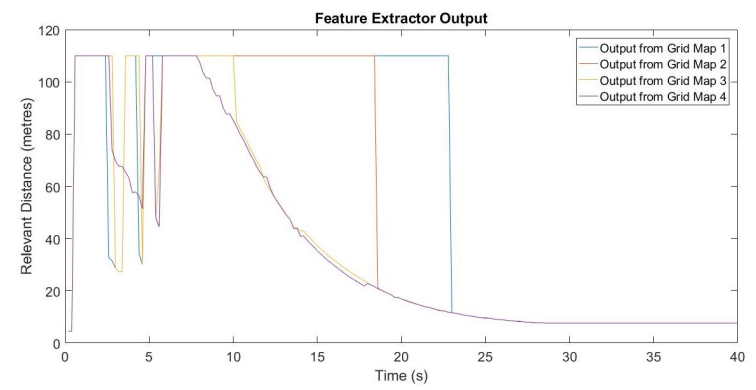

Fig. 9. Use Case 1: Relative distance outputs from Feature Extractor 


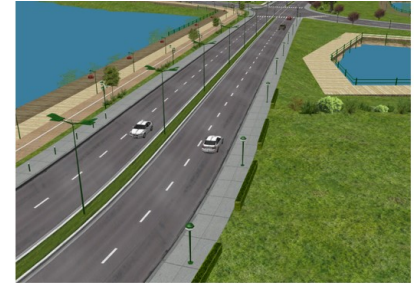

(a)

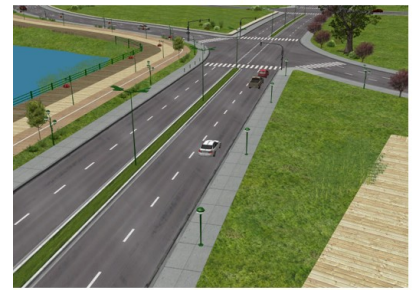

(c)

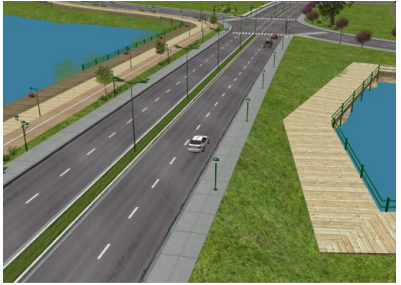

(b)

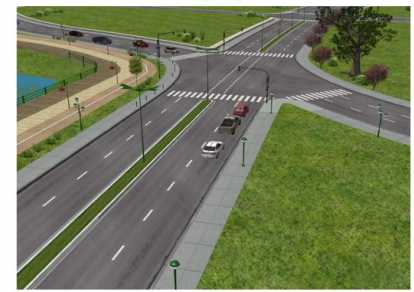

(d)
Fig. 7. Use Case 1: Snap shots of the test scenario in the order from ' $a$ ' to 'd'

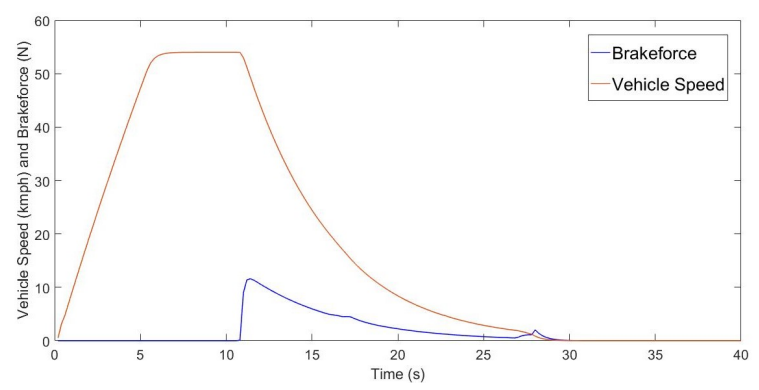

Fig. 8. Use Case 1: Profiles of vehicle speed and brake pedal force

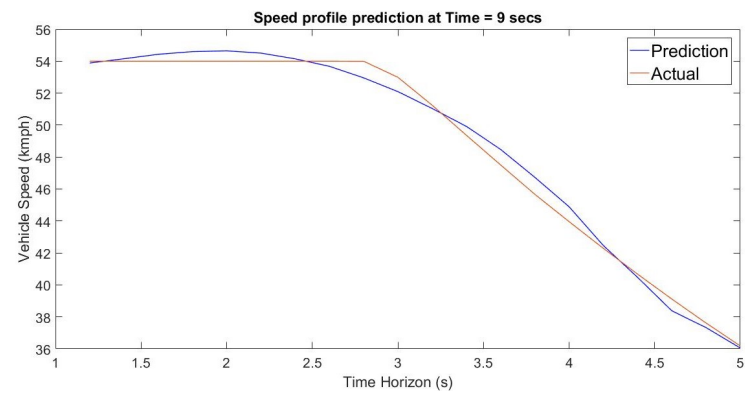

Fig. 10. Use Case 1: Vehicle Speed Profile prediction at time $=9$ secs

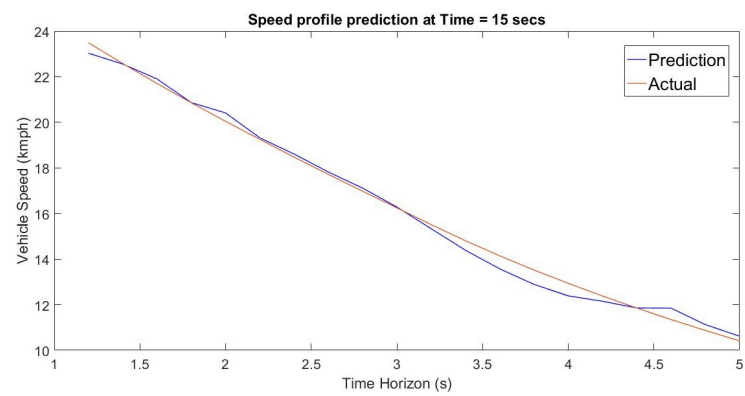

Fig. 11. Use Case 1: Vehicle Speed Profile prediction at time $=15 \mathrm{secs}$
Use Case 2: In this use case, the target vehicle overtakes a dynamic obstacle and comes back to its previous lane. To add complexity, another dynamic vehicle is present in the same lane which overtakes the same obstacle before the target vehicle. Hence, the human driver has to wait before overtaking the obstacle. The snapshots of the scenario are shown in Fig. 12. The vehicle speed, brake pedal force and the steering wheel angle profiles are shown in Fig. 13 and 14 respectively.

Similar to the previous use cases, the critical time instants considered from the point of view of vehicle speed prediction are 5.5 and 26 secs. The critical time instant for the steering wheel angle prediction considered is 15 secs. The model predictions have less accuracy as compared to the previous use cases due to the complex nature of driving in this scenario as shown in Fig. 15, 16 and 17. The model predicts the future profile (output) in the form of samples. During the prediction, the neural network does not consider these samples to be correlated and predicts them independently. Hence, polynomial regression (quadratic) can be performed to these predicted future samples (for both vehicle speed and steering wheel angle) to increase the prediction accuracy. This phenomenon is shown in the Fig. 15, 16 and 17. The regression is applied to the prediction only when the predicted outputs do not form a smooth profile. This polynomial regression is different than that applied in the model architecture for representing the past information of the inputs.

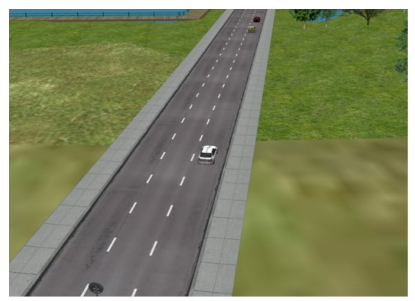

(a)

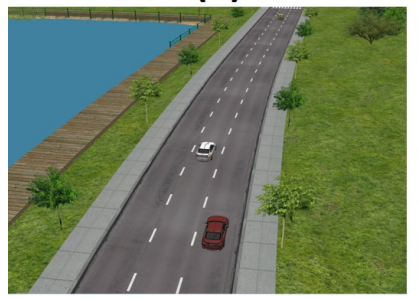

(c)

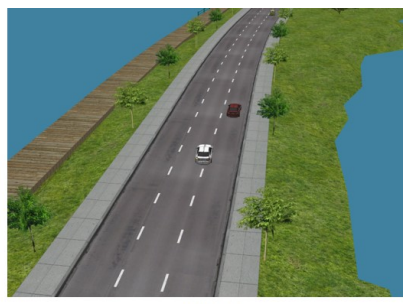

(b)

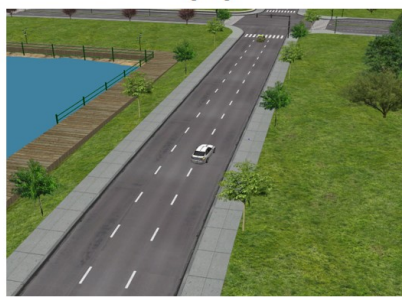

(d)
Fig. 12. Use Case 2: Snap shots of the test scenario in the order from 'a' to 'd' 


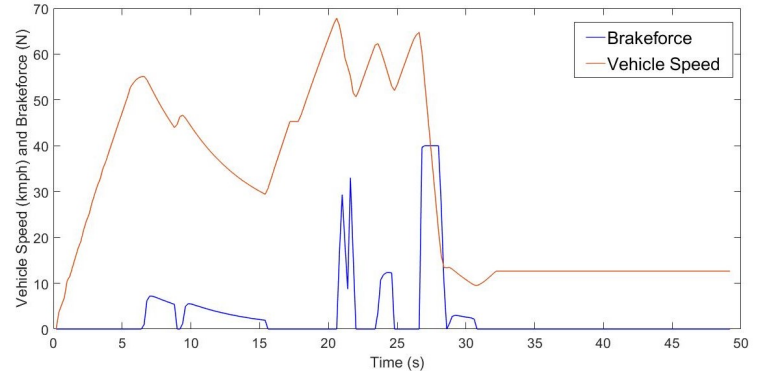

Fig. 13. Use Case 2: Profiles of vehicle speed and brake pedal force

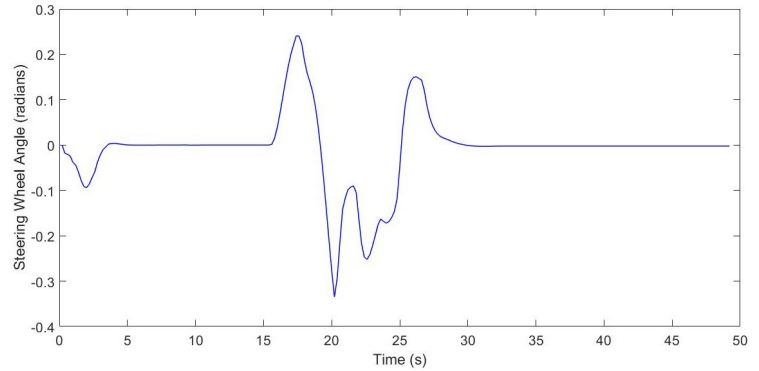

Fig. 14. Use Case 2: Steering wheel angle profile

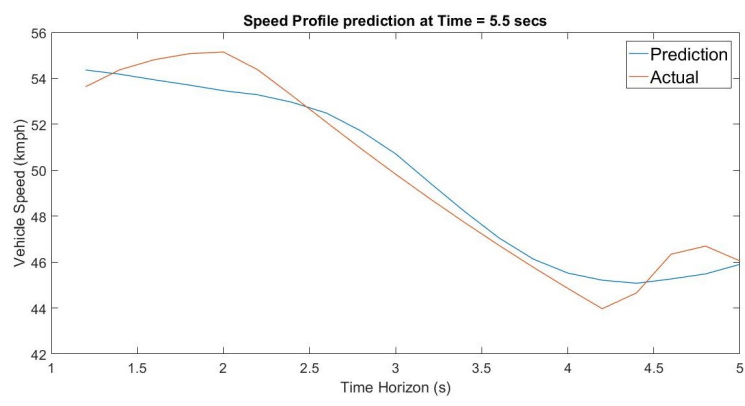

Fig. 15. Use Case 2: Vehicle Speed Profile prediction at time $=5.5$ secs

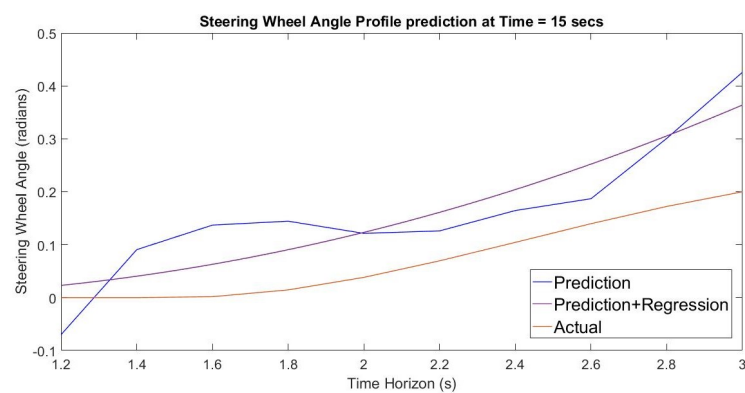

Fig. 16. Use Case 2: Steering Wheel Angle Profile prediction at time $=$ 15 secs

\section{CONCLusion And Future Works}

The presented model is developed with the objective of its application to the shared driving control methodology. Multilayer feed-forward neural network is used for the prediction of the human driving decisions (vehicle speed and steering wheel angle) for a particular time horizon. Environment

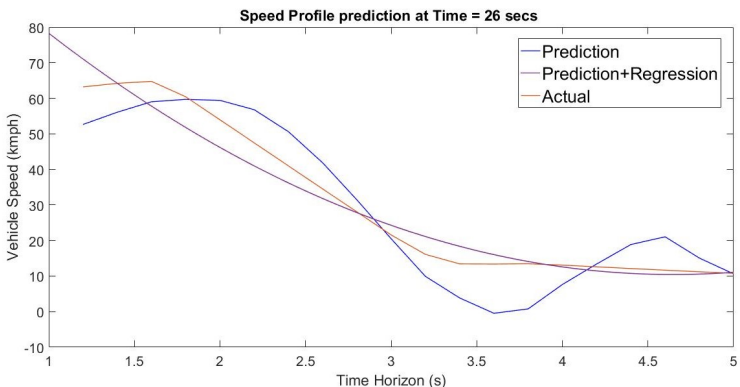

Fig. 17. Use Case 2: Vehicle Speed Profile prediction at time $=26$ secs

perception, vehicle state and human intention are majorly the three input categories considered in the methodology. The inputs related to the environment perception are derived from the LIDAR sensor data. Training and validation of the neural network is performed using the driving simulator setup and SCANeR studio software. The simulation results show that the model can accurately predict the human driving decisions for various scenarios. For the future works, the model is to be implemented in the real vehicle and validated with respect to the real vehicle data and applied to the shared driving control methodology.

\section{REFERENCES}

[1] S. Jugade, A. C. Victorino and V. B. Cherfaoui, "Human-Intelligent System Shared Control Strategy with Conflict Resolution," 2018 14th IEEE International Conference on Control and Automation, Anchorage, Alaska, USA, 2018.

[2] W. Takano, A. Matsushita, K. Iwao and Y. Nakamura, "Recognition of human driving behaviors based on stochastic symbolization of time series signal,” 2008 IEEE/RSJ International Conference on Intelligent Robots and Systems, Nice, 2008, pp. 167-172.

[3] L. Li, K. Ota and M. Dong, "Human-Like Driving: Empirical Decision-Making System for Autonomous Vehicles," in IEEE Transactions on Vehicular Technology.

[4] X. Meng, K. K. Lee and Y. Xu, "Human Driving Behavior Recognition Based on Hidden Markov Models," 2006 IEEE International Conference on Robotics and Biomimetics, Kunming, 2006, pp. 274 279.

[5] B. Kim, C. M. Kang, J. Kim, S. H. Lee, C. C. Chung and J. W. Choi, "Probabilistic vehicle trajectory prediction over occupancy grid map via recurrent neural network," 2017 IEEE 20th International Conference on Intelligent Transportation Systems (ITSC), Yokohama, 2017, pp. 399-404.

[6] F. Altch and A. de La Fortelle, "An LSTM network for highway trajectory prediction," 2017 IEEE 20th International Conference on Intelligent Transportation Systems (ITSC), Yokohama, 2017, pp. 353359.

[7] W. Yao, H. Zhao, P. Bonnifait and H. Zha, "Lane change trajectory prediction by using recorded human driving data," 2013 IEEE Intelligent Vehicles Symposium (IV), Gold Coast, QLD, 2013, pp. 430-436.

[8] A. Kuefler, J. Morton, T. Wheeler and M. Kochenderfer, "Imitating driver behavior with generative adversarial networks," 2017 IEEE Intelligent Vehicles Symposium (IV), Los Angeles, CA, 2017, pp. 204-211.

[9] C. Roesener, F. Fahrenkrog, A. Uhlig and L. Eckstein, "A scenariobased assessment approach for automated driving by using time series classification of human-driving behaviour," 2016 IEEE 19th International Conference on Intelligent Transportation Systems (ITSC), Rio de Janeiro, 2016, pp. 1360-1365.

[10] C. Zhao, J. Gong, C. Lu, G. Xiong and W. Mei, "Speed and steering angle prediction for intelligent vehicles based on deep belief network," 2017 IEEE 20th International Conference on Intelligent Transportation Systems (ITSC), Yokohama, 2017, pp. 301-306. 